Homology, Homotopy and Applications, vol.22(1), 2020, pp.215-232

\title{
ON TRIVIALITIES OF EULER CLASSES OF ORIENTED VECTOR BUNDLES OVER MANIFOLDS
}

\author{
ANIRUDDHA C. NAOLEKAR, B. SUBHASH AND AJAY SINGH THAKUR
}

(communicated by Donald M. Davis)

\begin{abstract}
We study manifolds on which all oriented bundles have trivial Euler class. In this note, we give a complete a characterization of such manifolds in dimension less than six, in terms of their cohomology groups, and obtain some partial results for manifolds of dimension 6 .
\end{abstract}

\section{Introduction}

One of the earliest known results on trivialities of characteristic classes of vector bundles was obtained by Atiyah-Hirzebruch by showing that the Stiefel-Whitney classes of any vector bundle over a 9-fold suspension $\Sigma^{9} X$ of a $C W$-complex $X$ are trivial [1, Theorem 2]. Further results on trivialities of Stiefel-Whitney classes of vector bundles over suspensions of $C W$-complexes have been studied in $[\mathbf{1 2}, \mathbf{1 6}, \mathbf{1 7}]$ and [18]. Similar results on trivialities of Chern classes of complex vector bundles over $C W$-complexes were studied in $[\mathbf{1 3}]$.

In recent times there has been some interest in trying to understand the set of Euler classes of oriented bundles over $C W$-complexes (see, for example, $[\mathbf{8}, \mathbf{9}, \mathbf{1 1}, \mathbf{1 4}, \mathbf{2 1}]$ ). In this note we are mainly interested in trivialities of Euler class of oriented vector bundles over manifolds.

We say that a closed connected smooth $n$-manifold $X$ has property $(\mathcal{E})$ (or satisfies $(\mathcal{E}))$ if for every oriented vector bundle $\alpha$ over $X$, the Euler class $e(\alpha)=0$.

Let $\mathcal{E}$ denote the set of diffeomorphism classes of closed connected smooth manifolds $X$ that have property $(\mathcal{E})$. Let $\mathcal{E}_{k}$ denote the subset of $\mathcal{E}$ consisting of manifolds $X$ with $\operatorname{dim} X=k$. In this note we shall completely describe the set $\mathcal{E}_{i}$ for $i \leqslant 5$ and obtain some necessary conditions for a closed connected smooth 6-manifold $X$ to satisfy $(\mathcal{E})$.

Recall that a smooth homology $n$-sphere is a closed connected smooth $n$-manifold $X$ such that $H_{i}(X ; \mathbb{Z}) \cong H_{i}\left(S^{n} ; \mathbb{Z}\right)$. If $X$ is a smooth homology $n$-sphere, where $n$ is odd and if $\alpha$ is a oriented vector bundle over $X$, then $e(\alpha)=0$ if $\operatorname{rank} \alpha \neq \operatorname{dim} X$. In the case when $\operatorname{rank} \alpha=\operatorname{dim} X$, we have $2 e(\alpha)=0$ which implies $e(\alpha)=0$. It follows that every smooth homology $n$-sphere where $n$ is odd has property $(\mathcal{E})$. The main results of this note are the following.

The research of third author is partially supported by DST-Inspire Faculty research grant (IFA-13MA-26).

Received December 28, 2018, revised July 8, 2019; published on November 20, 2019.

2010 Mathematics Subject Classification: 57R20.

Key words and phrases: Euler class.

Article available at http://dx.doi.org/10.4310/HHA.2020.v22.n1.a13

Copyright (C) 2019, International Press. Permission to copy for private use granted. 
Theorem 1.1. Let $X$ be a closed connected smooth 3-manifold. Then $X \in \mathcal{E}_{3}$ if and only if $X$ is a smooth homology 3-sphere.

It is easy to see (see Proposition 2.1 below) that an even dimensional closed connected orientable smooth manifold cannot have property $(\mathcal{E})$. In dimension 4 , the non-orientable closed connected smooth manifolds do not have property $(\mathcal{E})$ either. We prove the following.

Theorem 1.2. $\mathcal{E}_{4}=\emptyset$.

This is an immediate consequence of Theorem 3.2 below. The picture in dimension 5 is a bit more complicated. The following theorem completely describes the set $\mathcal{E}_{5}$.

Theorem 1.3. Let $X$ be a closed connected smooth 5-manifold. Then $X \in \mathcal{E}_{5}$ if and only if the following conditions are satisfied:

1. $H^{2}(X ; \mathbb{Z})=0, H^{4}(X ; \mathbb{Z})=0$, and

2. the 2-primary component of $H_{2}(X ; \mathbb{Z})$ is trivial.

Thus, if $X$ is a closed connected orientable smooth 5-manifold, then $X \in \mathcal{E}_{5}$ if and only if $\pi_{1}(X)$ is perfect and $X$ is a $\mathbb{Z}_{2}$-homology sphere (see Remark 4.1 below). In particular, the projective space $\mathbb{R P}^{5}$ and the Lens spaces in dimension 5 , do not satisfy $(\mathcal{E})$.

Using the Smale-Barden classification (see, $[\mathbf{1 5}, \mathbf{2}]$ ) of simply connected closed smooth 5-manifolds, we construct examples of simply connected closed smooth 5manifolds $X$ that satisfy $(\mathcal{E})$. We also construct examples of non-simply connected closed smooth orientable 5 -manifolds $X$ that satisfy $(\mathcal{E})$ and closed smooth nonorientable manifolds $X$ that satisfy $(\mathcal{E})$.

In dimension 6 we have not been able to derive a clear picture. We show, among other things, that $\mathcal{E}_{6}$ does not contain products and that any $X \in \mathcal{E}_{6}$ must have non-positive Euler characteristic.

Finally, we also compute the real and complex $K$-theory of $X \in \mathcal{E}_{5}$. As a consequence it follows that every orientable $X \in \mathcal{E}_{5}$ is stably parallelizable but not parallelizable since every such manifold has non-zero Kervaire semi-characteristic (see Remark 6.3(2) below).

This note is organized as follows. In Section 2 we collect some preliminary observations about the set $\mathcal{E}$, set up notations and state some results that we use for easy reference. In Section 3 we prove Theorem 1.1, Theorem 1.2 and state necessary conditions that any $X \in \mathcal{E}_{6}$ must satisfy. The proof of Theorem 1.3 is split into Sections 4 and 5 dealing with the orientable and non-orientable cases respectively. In Section 6 we compute the real and complex $K$-theory of $X \in \mathcal{E}_{5}$.

\section{Conventions}

Throughout, $F$ (respectively, $F^{\prime}, F^{\prime \prime}, \ldots$ ) will denote a finite abelian group. The integer $s$ (respectively, $s^{\prime}, s^{\prime \prime}, \ldots$ ) will denote the number of primes $p_{i}$ that are equal to 2 in a direct sum decomposition of $F$ (respectively, $F^{\prime}, F^{\prime \prime}, \ldots$ )

$$
F=\oplus_{i} \mathbb{Z} / p_{i}^{k_{i}}
$$

with the $p_{i}$ not necessarily distinct. Given $s$ (respectively, $s^{\prime}, s^{\prime \prime}, \ldots$ ), the pair of integers $u, v$ (respectively, $u^{\prime}, v^{\prime} ; u^{\prime \prime}, v^{\prime \prime} ; \ldots$ ) will denote the number of integers $k_{i}$ 
with $k_{i}=1$ and $k_{i}>1$ respectively. Thus $s=u+v$ (respectively, $s^{\prime}=u^{\prime}+v^{\prime} ; \ldots$ ). $\varepsilon^{k}$ will denote the trivial $k$-plane bundle. $\beta_{\mathbb{R}}$ will denote the underlying real bundle of a complex bundle $\beta$. In the sequel, a manifold will mean a closed, connected, smooth manifold.

\section{Preliminaries}

In this section we make some preliminary observations about the set $\mathcal{E}$. We begin with the following observation.

Proposition 2.1. Let $X$ be a manifold.

1. If $X$ is orientable and even dimensional, then $X \notin \mathcal{E}$.

2. If $X \in \mathcal{E}$, then $H^{2}(X ; \mathbb{Z})=0$.

3. If $X \in \mathcal{E}$, then the Steenrod square $S q^{1}: H^{1}\left(X ; \mathbb{Z}_{2}\right) \longrightarrow H^{2}\left(X ; \mathbb{Z}_{2}\right)$ is the zero homomorphism.

Proof. The first claim follows from $\left[\mathbf{1 1}\right.$, Theorem 2.4, (1)]. If $x \in H^{2}(X ; \mathbb{Z})$, then there exists a complex line bundle $\beta$ with $c_{1}(\beta)=e\left(\beta_{\mathbb{R}}\right)=x$. This proves (ii). To prove (iii), let $x \in H^{1}\left(X ; \mathbb{Z}_{2}\right)$ be non-zero and $\alpha$ a line bundle over $X$ with $w_{1}(\alpha)=x$. Then as

$$
S q^{1}(x)=x^{2}=w_{2}(\alpha \oplus \alpha)
$$

and $w_{2}(\alpha \oplus \alpha)$ is the mod-2 reduction of $e(\alpha \oplus \alpha)=0$, it follows that $S q^{1}(x)=0$. This proves (iii) and completes the proof of the proposition.

Lemma 2.2. Let $X, Y$ be orientable manifolds of positive dimension. Then $X \times Y \notin \mathcal{E}$.

Proof. If $X \times Y$ is even dimensional, then the previous proposition implies $X \times Y \notin$ $\mathcal{E}$. If $X \times Y$ is odd dimensional, we assume that $\operatorname{dim} X=2 n$ is even. Then there exists a $2 n$-plane bundle $\alpha$ over $X$ with $e(\alpha) \neq 0$. The projection $X \times Y \longrightarrow X$ induces a monomorphism in cohomology with integer coefficients in degree $2 n$. Thus the pullback of $\alpha$ to $X \times Y$ (by the projection) has non-zero Euler class.

Thus $\mathcal{E}$ does not contain orientable products. It is clear that $\mathcal{E}_{1}$ consists of the circle $S^{1}$. Since $H^{2}(X ; \mathbb{Z}) \neq 0$ for any 2-manifold, it follows that $\mathcal{E}_{2}=\emptyset$.

We now set up some notations. We shall make use of the following maps. Throughout, $\rho_{k}, \rho_{4,2}$ will denote the homomorphisms

$$
\begin{gathered}
\rho_{k}: H^{*}(X ; \mathbb{Z}) \longrightarrow H^{*}\left(X ; \mathbb{Z}_{k}\right), \\
\rho_{4,2}: H^{*}\left(X ; \mathbb{Z}_{4}\right) \longrightarrow H^{*}\left(X ; \mathbb{Z}_{2}\right)
\end{gathered}
$$

induced by the coefficient surjections. The homomorphism

$$
i_{*}: H^{*}\left(X ; \mathbb{Z}_{2}\right) \longrightarrow H^{*}\left(X ; \mathbb{Z}_{4}\right)
$$

is induced by the coefficient inclusion. Finally, recall that the Pontryagin square $\mathfrak{P}$ is a cohomology operation

$$
\mathfrak{P}: H^{2 k}\left(X ; \mathbb{Z}_{2}\right) \longrightarrow H^{4 k}\left(X ; \mathbb{Z}_{4}\right)
$$

with the property that

$$
\rho_{4,2} \mathfrak{P}(x)=x^{2} .
$$

It is well known (see, for example, $[\mathbf{1 9}$, Theorem $\mathrm{C}]$ ) that for any vector bundle $\alpha$ we 
have

$$
\mathfrak{P}\left(w_{2}(\alpha)\right)=\rho_{4}\left(p_{1}(\alpha)\right)+i_{*}\left(w_{1}(\alpha) S q^{1}\left(w_{2}(\alpha)\right)+w_{4}(\alpha)\right) .
$$

In particular, if either $\alpha$ is orientable or $w_{2}(\alpha)=0$ we have

$$
\mathfrak{P}\left(w_{2}(\alpha)\right)=\rho_{4}\left(p_{1}(\alpha)\right)+i_{*}\left(w_{4}(\alpha)\right) .
$$

The following observation will be crucially used in the proof of Theorem 1.3.

Proposition 2.3. Let $Y$ be a $C W$-complex. Assume that

$$
H_{i}(Y ; \mathbb{Z})= \begin{cases}\mathbb{Z} & i=0 \\ 0 & i=1, \\ F & i=2\end{cases}
$$

Further assume that $\mathbb{Z}_{2}$ is a direct summand in $H_{2}(Y ; \mathbb{Z})=F$. Then the Steenrod square $S q^{1}: H^{2}\left(Y ; \mathbb{Z}_{2}\right) \longrightarrow H^{3}\left(Y ; \mathbb{Z}_{2}\right)$ is non-zero.

Proof. Observe that

$$
H^{i}\left(Y ; \mathbb{Z}_{2}\right)=\left\{\begin{array}{cc}
0 & i=1, \\
\mathbb{Z}_{2}^{s} & i=2,
\end{array} \quad H^{i}\left(Y ; \mathbb{Z}_{4}\right)=\left\{\begin{array}{cc}
0 & i=1 \\
\mathbb{Z}_{2}^{u} \oplus \mathbb{Z}_{4}^{v} & i=2 .
\end{array}\right.\right.
$$

The conclusion now follows from the fact that $S q^{1}$ equals the Bockstein homomorphism associated to the exact sequence

$$
0 \longrightarrow Z_{2} \longrightarrow \mathbb{Z}_{4} \longrightarrow \mathbb{Z}_{2} \longrightarrow 0
$$

Indeed, we look at the following exact sequence

$$
0 \rightarrow H^{2}\left(Y ; \mathbb{Z}_{2}\right) \stackrel{i_{*}}{\longrightarrow} H^{2}\left(Y ; \mathbb{Z}_{4}\right) \stackrel{\rho_{4,2}}{\longrightarrow} H^{2}\left(Y ; \mathbb{Z}_{2}\right) \stackrel{S q^{1}}{\longrightarrow} H^{3}\left(Y ; \mathbb{Z}_{2}\right) \longrightarrow \cdots
$$

Identifying the individual groups in the above sequence, we get the exact sequence

$$
0 \rightarrow \mathbb{Z}_{2}^{u} \oplus \mathbb{Z}_{2}^{v} \stackrel{i_{*}}{\longrightarrow} \mathbb{Z}_{2}^{u} \oplus \mathbb{Z}_{4}^{v} \stackrel{\rho_{4,2}}{\longrightarrow} \mathbb{Z}_{2}^{u} \oplus \mathbb{Z}_{2}^{v} \stackrel{S q^{1}}{\longrightarrow} H^{3}\left(Y ; \mathbb{Z}_{2}\right) \rightarrow \cdots
$$

Notice that as $s=u+v$ and $u \neq 0$, it follows that $S q^{1}$ must be non-zero. This completes the proof.

Remark 2.4. We remark that the above proposition remains true even in the case that

$$
H_{i}(Y ; \mathbb{Z})= \begin{cases}\mathbb{Z} & i=0 \\ \mathbb{Z} & i=1 \\ F & i=2\end{cases}
$$

The proof is similar to the proof of the above proposition.

We end this section by stating some results for easy reference in a form that we shall use.

Theorem 2.5 ([5, Theorem 1]). Let $X$ be a connected $C W$-complex of dimension $\leqslant 5$. Let $a \in H^{2}\left(X ; \mathbb{Z}_{2}\right), b \in H^{4}\left(X ; \mathbb{Z}_{2}\right), c \in H^{4}(X ; \mathbb{Z})$ satisfy

$$
\rho_{4}(c)=\mathfrak{P}(a)+i_{*}(b) .
$$

Then there exists an oriented 5-plane bundle $\alpha$ such that $w_{2}(\alpha)=a, w_{4}(\alpha)=b$ and $p_{1}(\alpha)=c$. 
Theorem 2.6 ([5, Theorem 2]). Let $X$ be a connected $C W$-complex of dimension $\leqslant 5$. Let $a \in H^{2}\left(X ; \mathbb{Z}_{2}\right)$ and $p \in H^{4}(X ; \mathbb{Z})$ satisfy

$$
\rho_{4}(p)=\mathfrak{P}(a) .
$$

Then there exists an oriented 3-plane bundle $\alpha$ with $w_{2}(\alpha)=a$ and $p_{1}(\alpha)=p$.

Theorem 2.7 ([6, Theorem 2]). Let $X$ be a connected $C W$-complex of dimension $\leqslant 7$. Let $P, E \in H^{4}(X ; \mathbb{Z})$. Then there exists an oriented 4 -plane bundle $\alpha$ with

$$
w_{2}(\alpha)=0 ; \quad p_{1}(\alpha)=P ; \quad e(\alpha)=E
$$

if and only if there exists $U, V \in H^{4}(X ; \mathbb{Z})$ such that

1. $P=2 U$ and $E=2 V-U$,

2. $S q^{2} \rho_{2}(U)=0$ and $S q^{2} \rho_{2}(V)=0$,

3. $0 \in \Phi(U)$ and $0 \in \Phi(V)$,

where $\Phi$ is the secondary cohomology operation from $H^{4}(X ; \mathbb{Z})$ to $H^{7}\left(X ; \mathbb{Z}_{2}\right)$ associated to the relation $S q^{2} \circ S q^{2} \rho_{2}=0$.

\section{Dimensions 3, 4 and 6}

In this section we shall prove Theorem 1.1, Theorem 1.2 and obtain some necessary conditions on 6 -manifolds that satisfy $(\mathcal{E})$. We begin with the following observation that identifies the orientable manifolds in $\mathcal{E}_{3}$.

Proposition 3.1. Let $X$ be an orientable 3-manifold. Then $X \in \mathcal{E}_{3}$ if and only if $X$ is a homology 3-sphere.

Proof. Assume that $X \in \mathcal{E}_{3}$. By Proposition 2.1, (ii), we have $H^{2}(X ; \mathbb{Z})=0$. This implies that $H_{i}(X ; \mathbb{Z})=0, i=1,2$. Conversely, if $X$ is a homology 3 -sphere it follows from the comments in Section 1 that $X \in \mathcal{E}_{3}$.

To complete the proof of Theorem 1.1 we shall show that $\mathcal{E}_{3}$ does not contain any non-orientable manifold.

Proof of Theorem 1.1. Assume that there exists $X \in \mathcal{E}_{3}$ with $X$ non-orientable. Then, as $H^{2}(X ; \mathbb{Z})=0$, the integral and mod-2 cohomology groups of $X$ can be seen to be

$$
H^{i}(X ; \mathbb{Z})=\left\{\begin{array}{ll}
\mathbb{Z} & i=0, \\
\mathbb{Z} & i=1, \\
0 & i=2, \\
\mathbb{Z}_{2} & i=3,
\end{array} \quad H^{i}\left(X ; \mathbb{Z}_{2}\right)= \begin{cases}\mathbb{Z}_{2} & i=0, \\
\mathbb{Z}_{2} & i=1, \\
\mathbb{Z}_{2} & i=2, \\
\mathbb{Z}_{2} & i=3 .\end{cases}\right.
$$

By Remark 2.4, there exists $a \in H^{2}\left(X ; \mathbb{Z}_{2}\right)$ with $S q^{1}(a) \neq 0$. Clearly,

$$
\rho_{4}(p)=\mathfrak{P}(a)=0,
$$

where $0=p \in H^{4}(X ; \mathbb{Z})$. Hence, by Theorem 2.6, there exists an orientable 3-plane bundle $\alpha$ over $X$ with $w_{2}(\alpha)=a \neq 0$. Now,

$$
w_{3}(\alpha)=S q^{1}\left(w_{2}(\alpha)\right)=S q^{1}(a) \neq 0 .
$$

This implies $e(\alpha) \neq 0$ which is a contradiction. This completes the proof. 
We now turn to the proof of Theorem 1.2. We have already seen that $H^{2}(X ; \mathbb{Z})=0$ is a necessary condition for a manifold to satisfy $(\mathcal{E})$. In low dimensions, an additional necessary condition is the vanishing of the fourth integral cohomology. The precise statement is the following.

Theorem 3.2. Let $X$ be a n-manifold with $n=4,5$. If $X$ satisfies $(\mathcal{E})$, then $H^{4}(X ; \mathbb{Z})=0$.

Proof. Assume, if possible, that $H^{4}(X ; \mathbb{Z}) \neq 0$. Let $U \in H^{4}(X ; \mathbb{Z})$ with $U \neq 0$. Let $V=U, P=2 U$ and $E=2 U-U=U$. Then as all the three conditions of Theorem 2.7 are satisfied, there exists an oriented 4-plane bundle $\alpha$ with $e(\alpha)=U \neq 0$. This contradiction proves the theorem.

Proof of Theorem 1.2. If $X$ is a 4-manifold, then $H^{4}(X ; \mathbb{Z})$ equals either $\mathbb{Z}$ or $\mathbb{Z}_{2}$. Hence $\mathcal{E}_{4}=\emptyset$. This completes the proof of Theorem 1.2.

As a consequence of the above theorem we obtain the following observation.

Corollary 3.3. Suppose $X=X_{1} \times X_{2}$ is a non-orientable manifold, where $X_{1}, X_{2}$ are manifolds of positive dimension and $\operatorname{dim}(X)=5$. Then $X$ does not satisfy $(\mathcal{E})$.

Proof. If $\operatorname{dim} X_{1}=2$, then $H^{2}(X ; \mathbb{Z}) \neq 0$. If $\operatorname{dim} X_{1}=4$, then $H^{4}(X ; \mathbb{Z}) \neq 0$. In either case $X$ does not satisfy $(\mathcal{E})$.

We end this section by deriving some necessary conditions on manifolds $X \in \mathcal{E}_{6}$. We do not know if the conclusion of Theorem 3.2 remains true for 6 -manifolds. We can, however, make the following observation.

Theorem 3.4. Suppose $X \in \mathcal{E}_{6}$. Then

1. $H^{4}(X ; \mathbb{Z})$ is a finite elementary abelian 2-group, and

2. the Euler characteristic $\chi(X) \leqslant 0$.

Proof. Since $H^{4}\left(X ; \mathbb{Z}_{2}\right)$ is a finite elementary abelian 2-group the conclusion (1) follows if

$$
\rho_{2}: H^{4}(X ; \mathbb{Z}) \longrightarrow H^{4}\left(X ; \mathbb{Z}_{2}\right)
$$

is a monomorphism. If $U \neq 0$ and $U \in \operatorname{ker}\left(\rho_{2}\right)$ we set

$$
V=U, \quad E=U, \quad P=2 U .
$$

As $S q^{2} \rho_{2}(U)=0$ we have, by Theorem 2.7, an oriented 4-plane bundle $\alpha$ with

$$
e(\alpha)=U \neq 0 .
$$

This contradiction forces $\rho_{2}$ to be a monomorphism and (1) follows. In particular, $H^{4}(X ; \mathbb{Z})$ is finite. 
To prove (2), we note that, since $X$ is necessarily non-orientable, the integral homology of $X$ must now be of the form

$$
H_{i}(X ; \mathbb{Z})=\left\{\begin{array}{cc}
\mathbb{Z} & i=0, \\
\mathbb{Z}^{\ell} & i=1, \\
F & i=2, \\
\mathbb{Z}^{t} \oplus F^{\prime} & i=3, \\
F^{\prime \prime} & i=4, \\
\mathbb{Z}^{\ell^{\prime}} \oplus \mathbb{Z}_{2} & i=5, \\
0 & i=6,
\end{array}\right.
$$

where $F^{\prime}$ is a finite elementary abelian 2-group. The Euler characteristic can now be computed to be

$$
\chi(X)=1-\ell-t-\ell^{\prime} .
$$

Evidently $\ell \geqslant 1$ and so we must have $\chi(X) \leqslant 0$. This completes the proof.

We end this section by showing that a non-orientable 6 -dimensional product cannot satisfy $(\mathcal{E})$. Note that if a 6 -manifold $X$ satisfies $(\mathcal{E})$, then $X$ is necessarily nonorientable.

Proposition 3.5. Suppose $X=X_{1} \times X_{2}$ is a non-orientable manifold, where $X_{1}, X_{2}$ are manifolds of positive dimension and $\operatorname{dim}(X)=6$. Then $X$ does not satisfy $(\mathcal{E})$.

Proof. Assume that $X_{1}$ is non-orientable. We look at several cases. If $\operatorname{dim}\left(X_{1}\right)=2$, then $H^{2}(X ; \mathbb{Z}) \neq 0$ and hence $X$ does not satisfy $(\mathcal{E})$. If $\operatorname{dim}\left(X_{1}\right)=3$, then as $X_{1}$ is non-orientable there exists (by Theorem 1.1) an oriented bundle $\alpha$ over $X_{1}$ with nonzero Euler class. Pulling back $\alpha$ to $X$ via the projection $X \longrightarrow X_{1}$ gives an oriented bundle over $X$ with non-zero Euler class. Thus in this case too $X$ does not satisfy $(\mathcal{E})$. If $\operatorname{dim}\left(X_{1}\right)=4$, then arguing as in the previous case (and using Theorem 1.2) we see that $X$ does not satisfy $(\mathcal{E})$. Finally, let $\operatorname{dim}\left(X_{1}\right)=5$. Then first observe that $X_{2}=S^{1}$ and hence $H^{1}\left(X_{2} ; \mathbb{Z}\right)=\mathbb{Z}$. We now have two cases. In the case that $X_{1}$ does not satisfy $(\mathcal{E})$, then arguing as in the previous two cases we see that $X$ does not satisfy $(\mathcal{E})$. In the case that $X_{1}$ satisfies $(\mathcal{E})$, we have $H^{1}\left(X_{1} ; \mathbb{Z}\right)=\mathbb{Z}$ (see Section 5 ) and hence $H^{2}(X ; \mathbb{Z}) \neq 0$. Thus in this case too $X$ does not satisfy $(\mathcal{E})$. This completes the proof.

\section{Dimension 5: the orientable case}

In this section we shall prove Theorem 1.3 in the case $X$ is orientable.

Proof of Theorem 1.3. We assume that $X \in \mathcal{E}_{5}$ is orientable. We shall show that the conditions (1) and (2) of Theorem 1.3 are satisfied.

By Proposition 2.1 (ii) and Theorem 3.2 we have $H^{2}(X ; \mathbb{Z})=0$ and $H^{4}(X ; \mathbb{Z})=0$. Thus (1) is satisfied. To prove (2), we first observe that the integral homology and 
cohomology groups of $X$ can be seen to be

$$
H_{i}(X ; \mathbb{Z})=\left\{\begin{array}{ll}
\mathbb{Z} & i=0, \\
0 & i=1, \\
F & i=2, \\
0 & i=3, \\
0 & i=4, \\
\mathbb{Z} & i=5,
\end{array} \quad H^{i}(X ; \mathbb{Z})=\left\{\begin{array}{cc}
\mathbb{Z} & i=0, \\
0 & i=1, \\
0 & i=2, \\
F & i=3, \\
0 & i=4, \\
\mathbb{Z} & i=5 .
\end{array}\right.\right.
$$

where we recall that $F$ denotes a finite abelian group. Thus the mod-2 cohomology groups of $X$ are

$$
H^{i}\left(X ; \mathbb{Z}_{2}\right)=\left\{\begin{array}{cc}
\mathbb{Z}_{2} & i=0 \\
0 & i=1 \\
\mathbb{Z}_{2}^{s} & i=2 \\
\mathbb{Z}_{2}^{s} & i=3 \\
0 & i=4 \\
\mathbb{Z}_{2} & i=5
\end{array}\right.
$$

To complete the proof we shall show that $s=u+v=0$, where $u, v$ are as in the proof of Proposition 2.3. We first show that $u=0$.

Assume that $u>0$. We shall see that this leads to a contradiction. Since $u>0$, we conclude by Proposition 2.3 that

$$
S q^{1}: H^{2}\left(X ; \mathbb{Z}_{2}\right) \longrightarrow H^{3}\left(X ; \mathbb{Z}_{2}\right)
$$

is non-zero. Let $a \in H^{2}\left(X ; \mathbb{Z}_{2}\right)$ with $S q^{1}(a) \neq 0$. Since the Pontryagin square

$$
\mathfrak{P}: H^{2}\left(X ; \mathbb{Z}_{2}\right) \longrightarrow H^{4}\left(X ; \mathbb{Z}_{4}\right)=0
$$

is the zero homomorphism, we see that

$$
\rho_{4}(0)=\mathfrak{P}(a) .
$$

Thus, by Theorem 2.6, there exists an oriented 3-plane bundle $\alpha$ with $w_{2}(\alpha)=a \neq 0$. By Wu's formula we have

$$
w_{3}(\alpha)=S q^{1}\left(w_{2}(\alpha)\right)=S q^{1}(a) \neq 0 .
$$

This forces $e(\alpha) \neq 0$. This contradiction implies $u=0$.

Next we show that $v=0$. Assuming the contrary, that is, $v>0$ we shall construct a 5-dimensional $C W$-complex $X_{2}$ and a map $h: X \longrightarrow X_{2}$ such that

1. the homology groups of $X_{2}$ in degrees $\leqslant 2$ are of the form of the homology groups of $Y$ in Proposition 2.3,

2. $H_{2}\left(X_{2} ; \mathbb{Z}\right)=\mathbb{Z}_{2}$, and

3. $h^{*}: H^{3}\left(X_{2} ; \mathbb{Z}\right)=\mathbb{Z}^{\ell} \oplus \mathbb{Z}_{2} \longrightarrow H^{3}(X ; \mathbb{Z})=F$ is injective on the torsion subgroup.

We shall construct the space $X_{2}$ in two steps.

Step $I$. Let $X_{1}$ be the $C W$-complex obtained from $X$ by killing the fundamental group $\pi_{1}(X)$ of $X$. Let $f: X \longrightarrow X_{1}$ denote the inclusion. Since $X_{1}$ is obtained from $X$ by attaching 2-cells, we have $H_{i}\left(X_{1}\right)=H_{i}(X)$ for $i \neq 1,2$. As $X_{1}$ is simply connected, 
$H_{1}\left(X_{1} ; \mathbb{Z}\right)=0$. To compute $H_{2}\left(X_{1} ; \mathbb{Z}\right)$ we look at the exact sequence

$$
0 \longrightarrow H_{2}(X ; \mathbb{Z}) \stackrel{f_{*}}{\longrightarrow} H_{2}\left(X_{1}\right) \longrightarrow H_{2}\left(X_{1}, X ; \mathbb{Z}\right) \longrightarrow 0 .
$$

As $H_{2}\left(X_{1}, X ; \mathbb{Z}\right)=\mathbb{Z}^{t}$ (say) is free abelian, we have

$$
H_{2}\left(X_{1} ; \mathbb{Z}\right)=F \oplus \mathbb{Z}^{t}
$$

Thus the integral homology groups of $X_{1}$ are of the form

$$
H_{i}\left(X_{1} ; \mathbb{Z}\right)=\left\{\begin{array}{cl}
\mathbb{Z} & i=0, \\
0 & i=1, \\
\mathbb{Z}^{t} \oplus F & i=2, \\
0 & i=3, \\
0 & i=4, \\
\mathbb{Z} & i=5 .
\end{array}\right.
$$

In the exact sequence

$$
H^{3}\left(X_{1}, X ; \mathbb{Z}\right) \longrightarrow H^{3}\left(X_{1} ; \mathbb{Z}\right) \stackrel{f^{*}}{\longrightarrow} H^{3}(X ; \mathbb{Z}) \longrightarrow H^{4}\left(X_{1}, X ; \mathbb{Z}\right),
$$

the first and the fourth groups are zero and hence

$$
f^{*}: H^{3}\left(X_{1} ; \mathbb{Z}\right)=F \longrightarrow H^{3}(X ; \mathbb{Z})=F
$$

is an isomorphism.

Step II. From the above step, we have that $X_{1}$ is simply connected and $\pi_{2}\left(X_{1}\right)=$ $H_{2}\left(X_{1} ; \mathbb{Z}\right)=F \oplus \mathbb{Z}^{t}$. Since $v>0$, there exists an index two subgroup, $H$ say, of $F$. Let $X_{2}$ be obtained from $X_{1}$ by killing the index two subgroup $\mathbb{Z}^{t} \oplus H$ of $\pi_{2}\left(X_{1}\right)=$ $\mathbb{Z}^{t} \oplus F$. Let $g: X_{1} \longrightarrow X_{2}$ denote the inclusion and $h=g \circ f$. Note that

$$
g_{*}: H_{2}\left(X_{1} ; \mathbb{Z}\right)=F \oplus \mathbb{Z}^{t} \longrightarrow H_{2}\left(X_{2} ; \mathbb{Z}\right)=\mathbb{Z}_{2}
$$

is onto. Since we have an exact sequence

$$
0 \longrightarrow H_{3}\left(X_{2} ; \mathbb{Z}\right) \longrightarrow H_{3}\left(X_{2}, X_{1} ; \mathbb{Z}\right)
$$

and the last group is free abelian we conclude that $H_{3}\left(X_{2} ; \mathbb{Z}\right)=\mathbb{Z}^{\ell}$ (say) is free abelian. The integral homology groups of $X_{2}$ are now of the form

$$
H_{i}\left(X_{2} ; \mathbb{Z}\right)=\left\{\begin{array}{cl}
\mathbb{Z} & i=0, \\
0 & i=1, \\
\mathbb{Z}_{2} & i=2, \\
\mathbb{Z}^{\ell} & i=3, \\
0 & i=4, \\
\mathbb{Z} & i=5
\end{array}\right.
$$

Hence, by Proposition 2.3

$$
S q^{1}: H^{2}\left(X_{2} ; \mathbb{Z}_{2}\right)=\mathbb{Z}_{2} \longrightarrow H^{3}\left(X ; \mathbb{Z}_{2}\right)=\mathbb{Z}_{2}^{\ell} \oplus \mathbb{Z}_{2}
$$

is non-zero. We now argue that

$$
g^{*}: H^{3}\left(X_{2} ; \mathbb{Z}\right)=\mathbb{Z}^{\ell} \oplus \mathbb{Z}_{2} \longrightarrow H^{3}\left(X_{1} ; \mathbb{Z}\right)=F
$$


is injective on the torsion subgroup. This follows readily from the commutative diagram

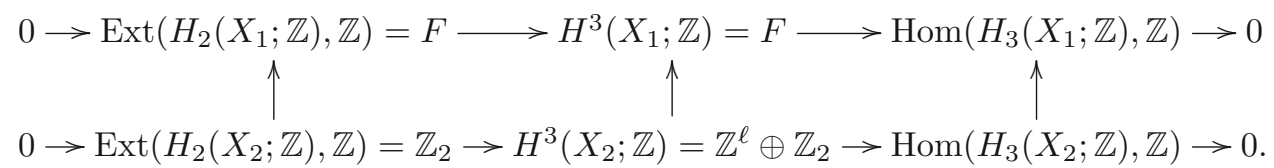

Observe that the first vertical map is a monomorphism, since $g_{*}: H_{2}\left(X_{1} ; \mathbb{Z}\right)=$ $F \oplus \mathbb{Z}^{t} \longrightarrow H_{2}\left(X_{2} ; \mathbb{Z}\right)=\mathbb{Z}_{2}$ is onto. The horizontal maps in the first square are monomorphisms. This forces

$$
g^{*}: H^{3}\left(X_{2} ; \mathbb{Z}\right)=\mathbb{Z}_{2} \oplus \mathbb{Z}^{\ell} \longrightarrow H^{3}\left(X_{1} ; \mathbb{Z}\right)=F
$$

to be injective on the torsion subgroup $\mathbb{Z}_{2}$ of $H^{3}\left(X_{2} ; \mathbb{Z}\right)$.

Now observe that as $H^{4}\left(X_{2} ; \mathbb{Z}_{4}\right)=0$, the Pontryagin square

$$
\mathfrak{P}: H^{2}\left(X_{2} ; \mathbb{Z}_{2}\right) \longrightarrow H^{4}\left(X_{2} ; \mathbb{Z}_{4}\right)
$$

is the zero map. We may now conclude, as before, that there exists an orientable 3 -plane bundle $\alpha$ over $X_{2}$ with $e(\alpha) \neq 0$. As $2 e(\alpha)=0$, we have that $e(\alpha)$ is of order two in $H^{3}\left(X_{2} ; \mathbb{Z}\right)=\mathbb{Z}^{\ell} \oplus \mathbb{Z}_{2}$. Hence $e\left(h^{*}(\alpha)\right) \neq 0$. This contradiction proves $v=0$ and hence (2) holds.

Conversely, if the two conditions of the theorem are satisfied and $\alpha$ is an oriented $k$-plane bundle over $X$ with $k=3$ or 5 , then as the integral cohomology groups are as described above and $2 e(\alpha)=0$ it readily follows that $e(\alpha)=0$. Hence $X \in \mathcal{E}_{5}$. This completes the proof of the theorem.

Remark 4.1. We remark that if $X$ is an orientable 5-manifold, then $X$ satisfies the two conditions in Theorem 1.3 if and only if $X$ satisfies either of the following two sets of equivalent conditions:

1. $\pi_{1}(X)$ is perfect,

2. $X$ is a rational homology sphere, and

3. the 2-primary component of $H_{2}(X ; \mathbb{Z})$ is trivial.

and

1. $\pi_{1}(X)$ is perfect, and

2. $X$ is a $\mathbb{Z}_{2}$-homology sphere.

We now make the following observations.

Corollary 4.2. If $X \in \mathcal{E}_{5}$ is orientable and $\alpha$ a vector bundle over $X$, then $w(\alpha)=1$. Also, $X$ is an oriented boundary.

Proof. We may assume that $\alpha$ is a 5 -plane bundle. By the remark above, $X$ is a $\mathbb{Z}_{2}$-homology sphere and hence $w_{i}(\alpha)=0$ for $1 \leqslant i<5$. Since $e(\alpha)=0$ we have $w_{5}(\alpha)=0$. This completes the proof.

In particular, $X$ is oriented cobordant to the sphere $S^{5}$. This is clearly not a sufficient condition for an orientable 5 -manifold to satisfy $(\mathcal{E})$. Indeed, the projective space $\mathbb{R} P^{5}$ is oriented cobordant to $S^{5}$ but does not satisfy $(\mathcal{E})$. It is well known that 
$\Omega_{5}^{S O} \cong \mathbb{Z}_{2}$ is generated by the Wu manifold $W=S U(3) / S O(3)$. The Wu manifold $W$ is a simply connected rational homology 5 -sphere with $H_{2}(X ; \mathbb{Z}) \cong \mathbb{Z}_{2}$. Hence $W$ does not satisfy $(\mathcal{E})$.

Suppose $X, Y \in \mathcal{E}_{5}$ are both orientable. It is then straightforward to check that the connected sum $X \# Y$ satisfies the two conditions of Theorem 1.3. Thus we have the following.

Corollary 4.3. Suppose $X, Y \in \mathcal{E}_{5}$ with both $X$ and $Y$ orientable. Then their connected sum $X \# Y \in \mathcal{E}_{5}$.

We now give examples of $X \in \mathcal{E}_{5}$ with $X$ orientable and not simply connected.

Example 4.4. Let $G$ be a finitely presented perfect group with trivial Schur multiplier. Then by $[\mathbf{1 0}$, Theorem 1], there exists an integral homology 5 -sphere $K$ with $\pi_{1}(K)=G$. Let $F$ be a finite abelian group with trivial 2-primary component. Then, by the Smale-Barden theory, $[\mathbf{1 5}, \mathbf{2}]$, there exists a simply connected 5-manifold $M$ with $H_{2}(M ; \mathbb{Z})=F \oplus F$. It is clear that $K, M \in \mathcal{E}_{5}$. As observed above, we have that $K \# M \in \mathcal{E}_{5}$. In particular, $\mathcal{E}_{5}$ contains non-simply connected orientable manifolds.

Given a connected $C W$-complex $Y$, there exists an exact sequence

$$
\pi_{2}(Y) \longrightarrow H_{2}(Y ; \mathbb{Z}) \longrightarrow H_{2}\left(\pi_{1}(Y) ; \mathbb{Z}\right) \longrightarrow 0
$$

(see, for example, [4, Theorem 5.2]) where, by definition, the last group is the Schur multiplier of $\pi_{1}(Y)$. Since $H_{2}(X ; \mathbb{Z})$ is finite for every orientable $X \in \mathcal{E}_{5}$ it follows that the Schur multiplier of $\pi_{1}(X)$ is finite for every orientable $X \in \mathcal{E}_{5}$ and does not contain any element of order 2 . It would be interesting to know if the fundamental group of any orientable $X \in \mathcal{E}_{5}$ has trivial Schur multiplier.

\section{Dimension 5: the non-orientable case}

In this section we prove Theorem 1.3 when $X$ is non-orientable, thereby completing the proof of Theorem 1.3. We begin with some observations.

Let $X$ be a non-orientable 5 -manifold with $H^{2}(X ; \mathbb{Z})=0$. Then the integral homology and cohomology groups of $X$ are of the form below

$$
H_{i}(X ; \mathbb{Z})=\left\{\begin{array}{cl}
\mathbb{Z} & i=0, \\
\mathbb{Z}^{\ell} & i=1, \\
F & i=2, \\
\mathbb{Z}^{t} \oplus F^{\prime} & i=3, \\
\mathbb{Z}^{\ell^{\prime}} \oplus \mathbb{Z}_{2} & i=4, \\
0 & i=5,
\end{array} \quad H^{i}(X ; \mathbb{Z})=\left\{\begin{array}{cc}
\mathbb{Z} & i=0, \\
\mathbb{Z}^{\ell} & i=1, \\
0 & i=2, \\
\mathbb{Z}^{t} \oplus F & i=3, \\
\mathbb{Z}^{\ell^{\prime}} \oplus F^{\prime} & i=4, \\
\mathbb{Z}_{2} & i=5 .
\end{array}\right.\right.
$$

Thus we have,

$$
H^{i}\left(X ; \mathbb{Z}_{2}\right)=\left\{\begin{array}{cc}
\mathbb{Z}_{2} & i=0 \\
\mathbb{Z}_{2}^{\ell} & i=1 \\
\mathbb{Z}_{2}^{s} & i=2, \\
\mathbb{Z}_{2}^{t} \oplus \mathbb{Z}_{2}^{s^{\prime}} \oplus \mathbb{Z}_{2}^{s} & i=3 \\
\mathbb{Z}_{2}^{\ell^{\prime}} \oplus \mathbb{Z}_{2} \oplus \mathbb{Z}_{2}^{s^{\prime}} & i=4 \\
\mathbb{Z}_{2} & i=5
\end{array}\right.
$$


By Poincaré duality we have

$$
\ell=\ell^{\prime}+1+s^{\prime}
$$

and

$$
s=t+s^{\prime}+s .
$$

Hence $t+s^{\prime}=0$. This forces $t=0=s^{\prime}$ and $\ell=\ell^{\prime}+1$. In particular, $\ell \geqslant 1$ (compare proof of Proposition 3.5).

If further $H^{4}(X ; \mathbb{Z})=0$, then the integral homology and cohomology groups of $X$ take the following form

$$
H_{i}(X ; \mathbb{Z})=\left\{\begin{array}{cl}
\mathbb{Z} & i=0, \\
\mathbb{Z} & i=1, \\
F & i=2, \\
0 & i=3, \\
\mathbb{Z}_{2} & i=4, \\
0 & i=5,
\end{array} \quad H^{i}(X ; \mathbb{Z})=\left\{\begin{array}{cc}
\mathbb{Z} & i=0, \\
\mathbb{Z} & i=1, \\
0 & i=2, \\
F & i=3, \\
0 & i=4, \\
\mathbb{Z}_{2} & i=5 .
\end{array}\right.\right.
$$

and hence we have

$$
H^{i}\left(X ; \mathbb{Z}_{2}\right)= \begin{cases}\mathbb{Z}_{2} & i=0 \\ \mathbb{Z}_{2} & i=1 \\ \mathbb{Z}_{2}^{s} & i=2 \\ \mathbb{Z}_{2}^{s} & i=3 \\ \mathbb{Z}_{2} & i=4 \\ \mathbb{Z}_{2} & i=5\end{cases}
$$

Before starting the proof of Theorem 1.3 we make some observations.

Lemma 5.1. Let $X$ be a non-orientable 5-manifold such that $H^{2}(X ; \mathbb{Z})=0$ and $H^{4}(X ; \mathbb{Z})=0$. Then the homomorphisms $S q^{1}: H^{4}\left(X ; \mathbb{Z}_{2}\right)=\mathbb{Z}_{2} \longrightarrow H^{5}\left(X ; \mathbb{Z}_{2}\right)=\mathbb{Z}_{2}$ and $i_{*}: H^{4}\left(X ; \mathbb{Z}_{2}\right)=\mathbb{Z}_{2} \longrightarrow H^{4}\left(X ; \mathbb{Z}_{4}\right)=\mathbb{Z}_{2}$ are isomorphisms.

Proof. Consider the long exact sequence

$$
\begin{aligned}
\cdots & \rightarrow H^{4}\left(X ; \mathbb{Z}_{2}\right) \stackrel{i^{*}}{\rightarrow} H^{4}\left(X ; \mathbb{Z}_{4}\right) \rightarrow H^{4}\left(X ; \mathbb{Z}_{2}\right) \stackrel{S q^{1}}{\rightarrow} H^{5}\left(X ; \mathbb{Z}_{2}\right) \\
& \rightarrow H^{5}\left(X ; \mathbb{Z}_{4}\right) \rightarrow H^{5}\left(X ; \mathbb{Z}_{2}\right) \rightarrow 0
\end{aligned}
$$

corresponding to the short exact sequence

$$
0 \longrightarrow \mathbb{Z}_{2} \longrightarrow \mathbb{Z}_{4} \longrightarrow \mathbb{Z}_{2} \longrightarrow 0
$$

of coefficient groups. Note that $H^{i}\left(X, \mathbb{Z}_{2}\right)=\mathbb{Z}_{2}$ and $H^{i}\left(X, \mathbb{Z}_{4}\right)=\mathbb{Z}_{2}$ for $i=4,5$, hence it follows that $i^{*}$ and $S q^{1}$ are isomorphisms.

Lemma 5.2. Let $X$ be a non-orientable 5-manifold with $H^{2}(X ; \mathbb{Z})=0, H^{4}(X ; \mathbb{Z})=$ 0 and $H^{2}\left(X ; \mathbb{Z}_{2}\right)=0$. Let $\alpha$ be an oriented 5 -plane bundle over $X$. Then $e(\alpha)=0$. 
Proof. We note that

$$
S q^{1}\left(w_{4}(\alpha)\right)=w_{1}(\alpha) w_{4}(\alpha)+w_{5}(\alpha)=w_{5}(\alpha)
$$

as $\alpha$ is orientable. As $w_{2}(\alpha)=0$ and $p_{1}(\alpha)=0$ we have

$$
0=\mathfrak{P}\left(w_{2}(\alpha)\right)=\rho_{4}\left(p_{1}(\alpha)\right)+i_{*}\left(w_{4}(\alpha)\right)=i_{*}\left(w_{4}(\alpha)\right) .
$$

Thus $w_{4}(\alpha)=0$ which implies that $w_{5}(\alpha)=0$ and hence $e(\alpha)=0$.

Proof of Theorem 1.3. Assume that $X \in \mathcal{E}_{5}$ is non-orientable. Then, by Proposition 2.1 and Theorem $3.2, H^{i}(X ; \mathbb{Z})=0$ for $i=2,4$ and hence (1) is satisfied. Observe that the homology and cohomology groups of $X$ are as noted above. To prove (2) we shall show, as in the orientable case, that $s=u+v=0$. We first show that $u=0$.

Assume that $u>0$. We shall see that this leads to a contradiction. By the remark after Proposition 2.3 we have that

$$
S q^{1}: H^{2}\left(X ; \mathbb{Z}_{2}\right)=\mathbb{Z}_{2}^{s} \longrightarrow H^{3}\left(X ; \mathbb{Z}_{2}\right)=\mathbb{Z}_{2}^{s}
$$

is non-zero. Hence there exists $a \in H^{2}\left(X ; \mathbb{Z}_{2}\right)$ with $S q^{1}(a) \neq 0$. Also note that $H^{4}(X ; \mathbb{Z})=0$. We now consider the Pontryagin square

$$
\mathfrak{P}: H^{2}\left(X ; \mathbb{Z}_{2}\right)=\mathbb{Z}_{2}^{s} \longrightarrow H^{4}\left(X ; \mathbb{Z}_{4}\right)=\mathbb{Z}_{2} \text {. }
$$

If $\mathfrak{P}(a)=0$, then as in the orientable case we get an oriented 3-plane bundle $\alpha$ over $X$ with $w_{2}(\alpha)=a$. This implies as before that $w_{3}(\alpha) \neq 0$ and hence $e(\alpha) \neq 0$ which is a contradiction. In the case that $\mathfrak{P}(a) \neq 0$ we can find $b \in H^{4}\left(X ; \mathbb{Z}_{2}\right)=\mathbb{Z}_{2}, b \neq 0$ with $i_{*}(b)=\mathfrak{P}(a)$. By Theorem 2.5, there exists an oriented 5 -plane bundle $\alpha$ with $w_{4}(\alpha)=b \neq 0$. By Lemma 5.1 ,

$$
w_{5}(\alpha)=S q^{1}\left(w_{4}(\alpha)\right) \neq 0
$$

and hence $e(\alpha) \neq 0$. This contradiction forces $u=0$.

We now show that $v=0$. We assume that $v>0$ and obtain a contradiction. The proof proceeds as in the orientable case and the case above. As before we construct spaces $X_{1}$ and $X_{2}$ where $X_{1}$ is obtained from $X$ by killing $\pi_{1}(X)$ and $X_{2}$ is obtained from $X_{1}$ by killing an index two subgroup of $\pi_{2}\left(X_{1}\right)=H_{2}\left(X_{1} ; \mathbb{Z}\right)=\mathbb{Z}^{t} \oplus F$. Let $f: X \longrightarrow X_{1}$ and $g: X_{1} \longrightarrow X_{2}$ denote the inclusion with $h=g \circ f$. As in the orientable case it can now be checked that

1. $h^{*}: H^{3}\left(X_{2} ; \mathbb{Z}\right)=\mathbb{Z}^{\ell} \oplus \mathbb{Z}_{2} \longrightarrow H^{3}(X ; \mathbb{Z})=F$ is injective on the torsion subgroup, and

2. $h^{*}: H^{5}\left(X_{2} ; \mathbb{Z}\right)=\mathbb{Z}_{2} \longrightarrow H^{5}(X ; \mathbb{Z})=\mathbb{Z}_{2}$ is an isomorphism.

Since $X_{2}$ is obtained from $X$ by attaching 2-cells and 3-cells we see that

$$
H^{4}\left(X_{2} ; \mathbb{Z}\right)=H^{4}\left(X_{1} ; \mathbb{Z}\right)=H^{4}(X ; \mathbb{Z})=0 .
$$

Also as $H_{2}\left(X_{2} ; \mathbb{Z}\right)=\mathbb{Z}_{2}$ we have

$$
S q^{1}: H^{2}\left(X_{2} ; \mathbb{Z}_{2}\right)=\mathbb{Z}_{2} \longrightarrow H^{3}\left(X_{2} ; \mathbb{Z}_{2}\right)
$$

is non-zero. As before we fix $a \in H^{2}\left(X_{2} ; \mathbb{Z}_{2}\right)$ with $S q^{1}(a) \neq 0$. If $\mathfrak{P}(a)=0$, then arguing as above we have an oriented 3 -plane bundle $\alpha$ with $e(\alpha) \neq 0$. This implies $h^{*}(e(\alpha)) \neq 0$. This is a contradiction. If $\mathfrak{P}(a) \neq 0$, then we have an oriented 5 -plane 
bundle with $e(\alpha) \neq 0$. Hence $h^{*}(e(\alpha)) \neq 0$ and we have a contradiction. This forces $v=0$. This completes the proof of the theorem in one direction.

Conversely, assume that $X$ is non-orientable and satisfies the two conditions of the theorem. In particular $s=0$. Now if $\alpha$ is an oriented 3-plane bundle then clearly $e(\alpha)=0$. If $\alpha$ is an oriented 5-plane bundle, then by Lemma 5.2 we have $e(\alpha)=0$. Hence $X \in \mathcal{E}_{5}$. This completes the proof of the theorem.

As in the orientable case we have the following:

Corollary 5.3. If $X \in \mathcal{E}_{5}$ is non-orientable and $\alpha$ a vector bundle over $X$, then

1. $w(\alpha)=1$ if $\alpha$ is orientable, and

2. $w_{i}(\alpha)=0, i \geqslant 2$, if $\alpha$ is non-orientable.

In particular, $X$ is an unoriented boundary.

Proof. We first prove (1). It is enough to prove this when $\alpha$ is an orientable 5 -plane bundle. Since $e(\alpha)=0$ we have $w_{5}(\alpha)=0$. The equality

$$
S q^{1}\left(w_{4}(\alpha)\right)=w_{5}(\alpha)=0,
$$

together with the fact that $S q^{1}$ is an isomorphism (Lemma 5.1) implies that $w_{4}(\alpha)=$ 0 . Since $H^{2}\left(X ; \mathbb{Z}_{2}\right)=0$ we have $w_{2}(\alpha)=0$. That $w_{1}(\alpha)=0$ follows from the assumption that $\alpha$ is orientable. This forces $w_{3}(\alpha)=0$ as the first non-zero Stiefel-Whitney class must appear in a degree a power of 2 . This proves (1).

Next we prove (2). Assume that $\alpha$ is non-orientable. We assume as before that $\alpha$ is a 5 -plane bundle. As $H^{2}\left(X ; \mathbb{Z}_{2}\right)=0$ and

$$
S q^{1}\left(w_{2}(\alpha)\right)=w_{3}(\alpha)
$$

we have $w_{i}(\alpha)=0, i=2,3$. We next show that $w_{5}(\alpha)=0$. Assume $w_{5}(\alpha) \neq 0$. Now

$$
S q^{1}\left(w_{4}(\alpha)\right)=w_{1}(\alpha) w_{4}(\alpha)+w_{5}(\alpha) .
$$

If $w_{4}(\alpha)=0$, then $0=S q^{1}\left(w_{4}(\alpha)\right)=w_{5}(\alpha)$ is a contradiction. On the other hand assuming $w_{4}(\alpha) \neq 0$ we have

$$
S q^{1}\left(w_{4}(\alpha)\right)=w_{1}(\alpha) w_{4}(\alpha)+w_{5}(\alpha)=0
$$

which is a contradiction since $S q^{1}$ is an isomorphism, by Lemma 5.1. Thus $w_{5}(\alpha)=0$. Finally, we show that $w_{4}(\alpha)=0$. This follows from the fact that

$$
i_{*}\left(w_{4}(\alpha)\right)+\rho_{4}\left(p_{1}(\alpha)\right)=\mathfrak{P}\left(w_{2}(\alpha)\right)=0
$$

implying that $i_{*}\left(w_{4}(\alpha)\right)=0$. This implies $w_{4}(\alpha)=0$. This completes the proof of (2).

Suppose $X, Y \in \mathcal{E}_{5}$ where at least one of $X$ and $Y$ is orientable. Then it is easy to check that the connected sum $X \# Y$ satisfies the two conditions of Theorem 1.3. Thus we have the following.

Corollary 5.4. Suppose $X, Y \in \mathcal{E}_{5}$ where at least one of $X$ and $Y$ is orientable. Then $X \# Y \in \mathcal{E}_{5}$.

We mention that if $X, Y \in \mathcal{E}_{5}$ are both non-orientable, then as $H_{1}(X \# Y ; \mathbb{Z})=$ $\mathbb{Z} \oplus \mathbb{Z}$, it follows that $X \# Y$ does not satisfy $(\mathcal{E})$. We end this section by giving examples of non-orientable 5 -manifolds that satisfy $(\mathcal{E})$. 
Example 5.5. Let $E=S^{4} \widetilde{\times} S^{1}$ denote the twisted $S^{4}$ bundle over $S^{1}$. The space $E$ is obtained as the quotient

$$
E=S^{4} \times[0,1] / \sim
$$

where $(x, 0) \sim(-x, 1)$. The integral homology groups of $E$ are of the form

$$
H_{i}(E ; \mathbb{Z})=\left\{\begin{array}{cl}
\mathbb{Z} & i=0 \\
\mathbb{Z} & i=1, \\
0 & i=2, \\
0 & i=3, \\
\mathbb{Z}_{2} & i=4 \\
0 & i=5
\end{array}\right.
$$

Thus, by Theorem 1.3, $E \in \mathcal{E}_{5}$. Observe that $E$ is non-orientable and $\pi_{1}(E)=\mathbb{Z}$. Let $M \in \mathcal{E}_{5}$ be an orientable manifold. Then $E \# M$ is non-orientable and $E \# M \in \mathcal{E}_{5}$.

Notice that if $X \in \mathcal{E}_{5}$ is non-orientable, then $\pi_{1}(X)$ is necessarily infinite. However, as $H_{2}(X ; \mathbb{Z})$ is finite, it follows that the Schur multiplier of $\pi_{1}(X)$ is still finite and has trivial 2-primary component.

\section{6. $\quad K$-theory}

In this section we describe the real and complex $K$-theory of manifolds $X \in \mathcal{E}_{5}$. Recall the Atiyah-Hirzebruch spectral sequence for $\widetilde{K}(X)$ is the spectral sequence with $E_{2}$ term given by

$$
E_{2}^{p, q}=\widetilde{H}^{p}\left(X ; K^{q}(\text { point })\right)
$$

Similarly the Atiyah-Hirzebruch spectral sequence for $\widetilde{K O}(X)$ is the spectral sequence with $E_{2}$ term given by

$$
E_{2}^{p, q}=\widetilde{H}^{p}\left(X ; K O^{q}(\text { point })\right) .
$$

We have the following description of the real and complex $K$-theory for an orientable $X \in \mathcal{E}_{5}$.

Theorem 6.1. Let $X \in \mathcal{E}_{5}$ be orientable with $H_{2}(X, \mathbb{Z})=F$.

1. $\widetilde{K O}^{-i}(X)$ is given as follows:

\begin{tabular}{|c|c|c|c|c|c|c|c|c|}
\hline$i$ & 0 & 1 & 2 & 3 & 4 & 5 & 6 & 7 \\
\hline$\widetilde{K O}^{-i}(X)$ & 0 & $F$ & 0 & $\mathbb{Z}$ & $\mathbb{Z}_{2}$ & $\mathbb{Z}_{2} \oplus F$ & 0 & $\mathbb{Z}$ \\
\hline
\end{tabular}

2. $\widetilde{K}^{0}(X)=0$ and $\widetilde{K}^{-1}(X)=\mathbb{Z} \oplus F$.

Proof. The Atiyah-Hirzebruch spectral sequence for both $\widetilde{K O}(X)$ and $\widetilde{K}(X)$ collapses after the sixth page, since all the differentials are zero. The proof of the theorem now follows easily by analyzing these spectral sequences and using the cohomology description of $X$ when $X \in \mathcal{E}_{5}$ and $X$ orientable, except for the value of $\widetilde{K}^{-1}(X)$. For the computation of $\widetilde{K}^{-1}(X)$, we consider the following long exact sequence (see 
p. 340 of $[\mathbf{2 0}])$,

$$
\cdots \rightarrow \widetilde{K O}^{0}(X) \rightarrow \widetilde{K O}^{-1}(X) \rightarrow \widetilde{K}^{-1}(X) \rightarrow \widetilde{K O}^{1}(X) \rightarrow \widetilde{K O}^{0}(X) \rightarrow \cdots .
$$

As $\widetilde{K O}^{0}(X)=0, \widetilde{K O}^{-1}(X)=F$ and $\widetilde{K O}^{1}(X)=\mathbb{Z}$, we conclude that $\widetilde{K}^{-1}(X)=$ $\mathbb{Z} \oplus F$

When $X \in \mathcal{E}_{5}$ is non-orientable, we have the following description of real and complex $K$-theory.

Theorem 6.2. Let $X \in \mathcal{E}_{5}$ be non-orientable with $H_{2}(X, \mathbb{Z})=F$. Then $\widetilde{K}^{0}(X)=0$ and $\widetilde{K}^{-1}(X)=\mathbb{Z} \oplus \mathbb{Z}_{2} \oplus F$. The KO-groups of $X$ is as follows:

\begin{tabular}{|c|c|c|c|c|c|c|c|c|}
\hline$i$ & 0 & 1 & 2 & 3 & 4 & 5 & 6 & 7 \\
\hline$\widetilde{K O}^{-i}(X)$ & $\mathbb{Z}_{2}$ & $\mathbb{Z}_{2} \oplus F$ & 0 & $\mathbb{Z} \oplus \mathbb{Z}_{2}$ & $\mathbb{Z}_{2}$ & $\mathbb{Z}_{2} \oplus \mathbb{Z}_{2} \oplus F$ or $\mathbb{Z}_{4} \oplus F$ & $\mathbb{Z}_{2}$ & $\mathbb{Z} \oplus \mathbb{Z}_{2}$ \\
\hline
\end{tabular}

Proof. As noted above, the Atiyah-Hirzebruch spectral sequences for $\widetilde{K}(X)$ and $\widetilde{K O}(X)$ collapses after the sixth page. The proof of the theorem now follows easily by further analyzing the spectral sequences, except for the value of $\widetilde{K O}^{0}(X)$ and $\widetilde{K O}^{-6}(X)$. From the analysis we observe that $\widetilde{K O}^{0}(X)$ is either 0 or $\mathbb{Z}_{2}$ and $\widetilde{K O}^{-6}(X)$ is either $\mathbb{Z}_{2}$ or 0 .

Since $X$ is non-orientable, the tangent bundle over $X$ will represent the non-zero element in $\widetilde{K O}^{0}(X)$. Hence $\widetilde{K O}^{0}(X) \cong \mathbb{Z}_{2}$.

If $\widetilde{K O}^{-6}(X)=0$ then we get the following short exact sequence (see p. 340 of [20])

$$
0 \rightarrow \widetilde{K O}^{-7}(X) \stackrel{\epsilon}{\rightarrow} \widetilde{K}^{-7}(X) \rightarrow \widetilde{K O}^{-5}(X) \rightarrow 0,
$$

where the first map $\epsilon$ is the complexification map. Then $\epsilon$ will map the $\mathbb{Z}_{2}$-summand of $\widetilde{K O}^{-7}(X)$ onto the $\mathbb{Z}_{2}$ summand of $\widetilde{K}^{-7}(X)$. If $\rho: \widetilde{K}^{-7}(X) \rightarrow \widetilde{K O}^{-7}(X)$ denotes the real restriction then using the fact that the composition $\rho \circ \epsilon$ is multiplication by 2 , it is easy to see that $\epsilon$ will map the $\mathbb{Z}$-summand of $\widetilde{K O}^{-7}(X)$ into the $\mathbb{Z}$-summand of $\widetilde{K}^{-7}(X)$ either isomorphically or by multiplication of 2 . Applying this to the above short exact sequence, we get the contradiction on the cardinality of the groups. Hence, $\widetilde{K O}^{-6}(X)=\mathbb{Z}_{2}$.

Remark 6.3. $\quad$ 1. It follows from the $K O$-theory computations that if $X \in \mathcal{E}_{5}$ is orientable, then $X$ is stably parallelizable. Thus, these $X$ provide examples of stably parallelizable $\mathbb{Z}_{2}$-homology 5 -spheres. On the other hand it is known that there exist $\mathbb{Z}_{2}$-homology 5 -spheres, for example the Lens space $L(5 ; 1,1,1)$ in the notation of $[\mathbf{7}]$, that are not stably parallelizable.

2. As noted above, if $X \in \mathcal{E}_{5}$ is orientable, then $X$ is stably parallelizable. The Kervaire semi-characteristic $\chi^{*}(X)$ of a $(2 n+1)$-dimensional manifold is given by (see $[\mathbf{3}]$ )

$$
\chi^{*}(X)=\sum_{i=0}^{n} \operatorname{dim} H^{i}\left(X ; \mathbb{Z}_{2}\right) \bmod 2 .
$$

If $X \in \mathcal{E}_{5}$ is orientable, then the computation of cohomology groups of $X \in \mathcal{E}_{5}$ in Theorem 1.3, shows that $\chi^{*}(X)=1$. This implies that if $X \in \mathcal{E}_{5}$ is orientable, 
then $X$ cannot be parallelizable by Theorem 1.2 from [3]. Moreover, in this case it follows that $\operatorname{span}(X)=1$.

\section{Acknowledgments}

The authors thank the anonymous referee for his detailed and valuable comments which has helped in improving the general presentation of the paper and correcting a few inaccuracies.

\section{References}

[1] Atiyah, M., and Hirzebruch, F., Bott periodicity and the parallelizability of the spheres, Proc. Cambridge Philos. Soc., 57 (1961), 223-226.

[2] Barden, D., Simply connected five-manifolds, Ann. of Math. (2), 82 (3) (1965), 365-385.

[3] Bredon, G.E., and Kosinski, A., Vector fields on $\pi$-manifolds, Ann. of Math., 84 (1) (1966), 85-90.

[4] Brown, K.S., Cohomology of Groups, Springer, Grad. Texts in Math., vol. 87, 1982.

[5] Čadek, M., and Vanžura, J., On the classification of oriented bundles over 5-complexes, Czechoslovak Math. J., 43 (118), no. 4, (1993), 753-764.

[6] Čadek, M., and Vanžura, J., On oriented vector bundles over $C W$-complexes of dimension 6 and 7, Comment. Math. Univ. Carolin., 33 (4) (1992), 727-736.

[7] Ewing, J., Moolgavkar, S., and Smith, L., Stable parallelizability of Lens spaces, J. Pure Appl. Algebra, 10 (1977), 177-191.

[8] Guijarro L., Schick T., and Walschap G., Bundles with spherical Euler class, Pacific J. Math., 27 (2) (2002), 377-392.

[9] Guijarro, L., and Walschap, G., Transitive holonomy group and rigidity in nonnegative curvature, Math. Z., 237 (2001), 265-281.

[10] Kervaire, Michel, A., Smooth homology spheres and their fundamental groups, Trans. Amer. Math. Soc., 144 (1969), 67-72

[11] Naolekar, A.C., Realizing cohomology classes as Euler classes, Math. Slovaca, 62 (5) (2012), 949-966.

[12] Naolekar, A.C., and Thakur, A.S., Vector bundles over iterated suspensions of stunted real projective spaces., Acta Math. Hungar., 142 (2014), 339-347.

[13] Naolekar, A.C., and Thakur, A.S., On trivialities of Chern classes, Acta Math. Hungar., 144 (1) (2014), 99-109.

[14] Naolekar, A.C., and Thakur, A.S., Euler classes of vector bundles over iterated suspensions of real projective spaces, Math. Slovaca, 68 (3) (2018), 677-684.

[15] Smale, S., On the structure of 5-manifolds, Ann. of Math., 75 (1) (1962), 38-46.

[16] Tanaka, R., On trivialities of Stiefel-Whitney classes of vector bundles over highly connected complexes, Topology Appl., 155 (2008), 1687-1693. 
[17] Tanaka, R., On trivialities of Stiefel-Whitney classes of vector bundles over iterated suspension spaces, Homology Homotopy Appl., 12 (1) (2010), 357-366.

[18] Thakur, A.S., On trivialities of Stiefel-Whitney classes of vector bundles over iterated suspensions of Dold manifolds, Homology Homotopy Appl., 15 (2013), 223-233.

[19] Thomas, E., On the cohomology of the real Grassmann complexes and the characteristic classes of n-plane bundles, Trans. Amer. Math. Soc., 96 (1) (1960), $67-89$.

[20] Toda, H., Order of the identity class of a suspension space, Ann. of Math., 78 (1963), 300-325.

[21] Walschap, G., The Euler class as a cohomology generator, Illinois J. Math., 46 (1) (2002), 165-169.

Aniruddha C. Naolekar ani@isibang.ac.in

Stat-Math Unit, Indian Statistical Institute, 8th Mile, Mysore Road, RVCE Post, Bangalore 560059, India

B. Subhash subhash@iisertirupati.ac.in

Indian Institute of Science Education and research (IISER) Tirupati, Tirupati 517507, India

Ajay Singh Thakur asthakur@iitk.ac.in

Department of Mathematics and Statistics, Indian Institute of Technology Kanpur, Kanpur 208016, India 